\title{
Analysis of the electrical characteristics of SCR-based ESD Protection Device (PTSCR) in 0.13/0.18/0.35 um process technology
}

\author{
Yong-Seo Koo ${ }^{\text {a) }}$ \\ Department of Electronics and Electrical Engineering Dankook University \\ 126, Jukjeon-dong, Suji-gu, Yongin-si, Gyeonggi-do, 448-701, Korea \\ a)yskoo@dankook.ac.kr
}

Abstract: In this paper, an experimental analysis of the electrical characteristics of the PTSCR was conducted. The PTSCR contains a high trigger current and a holding voltage that enables latch-up immune during normal operation. The PTSCR in each process technology is verified by the TLP system as well as a hot chuck controller. The experimental results show that the trigger current and holding voltage are higher than that of other SCR devices. At higher temperatures, the holding voltage of the PTSCR decreased due to the operation mechanism of the SCR, while the trigger current increased due to the MOSFET trigger mechanism.

Keywords: ESD, SCR, gate grounded NMOS (GGNMOS), holding voltage, trigger current

Classification: Electron devices, circuits, and systems

\section{References}

[1] Huang, et al., "ESD protection design for advanced CMOS," Proc. SPIE, pp. 123-131, 2001.

[2] O. Semenov, H. Sarbishaei, and M. Sachdev, ESD Protection Device and Circuit Design for Advanced CMOS Technologies, Springer, Netherlands, 2008.

[3] V. Vashchenko, A. Concannon, M. ter Beek, and P. Hopper, "High holding voltage cascoded LVTSCR structures for $5.5-\mathrm{V}$ tolerant ESD protection clamps," IEEE Trans. Device Mater. Rel., vol. 4, pp. 273-280, 2004.

[4] S. H. Voldman, ESD: Circuits and Devices. London, U.K.: Wiley, 2006.

[5] M. D. Ker and H. H. Chang, "How to safely apply the LVTSCR for CMOS whole-chip ESD protection without being accidentally triggered on," $J$. Electro- statics, vol. 47, pp. 215-248, 1999.

[6] Y. Koo, K. Lee, K. Kim, and J. Kwon, "Design of SCR-based ESD protection device for power clamp using deep-submicron CMOS technology," Microelectronics Journal, vol. 40, pp. 1007-1012, 2009.

[7] S.-L. Jang, L.-S. Lin, and S.-H. Li, "Temperature-dependent dynamic triggering characteristics of SCR-type ESD protection circuits," Solid-State Electronics, vol. 45, pp. 2005-2009, 2001. 


\section{Introduction}

For decades, electrostatic discharge has been considered a major reliability threat in the semiconductor industry. It was reported that ESD and EOS are responsible for up to $70 \%$ of the failure in IC technology $[1,2,3]$. Therefore, each Input/output power clamp must be designed with protection circuitry that creates a discharge path for the ESD current [4]. Silicon-controlled rectifier (SCR) devices have long been favored as the on-chip ESD protection elements for many applications because of their superior ESD behavior, possessing high failure currents and low on-resistances [5]. However, the SCR contains a high trigger voltage. Additionally, difficulty exists in effectively discharging ESD current before a malfunction of the core circuit occurs. The SCR also causes a transient-induced latch-up problem due to its lower holding voltage [6]. The latch-up problem in normal operation can be avoided if the PTSCR possessed a higher holding voltage and higher trigger current.

IC speed, chip size and circuit density increased with the advancements made in ULSI technology. This led to an increase in power dissipation within the IC as well as an increase in the overall operating temperature of the IC. Therefore, gaining a thorough understanding of the temperature dependency of an ESD protection device at high temperatures is imperative for developing a method for alleviating the increased power dissipation within the IC, as well as thwarting the possible thermal damage to the ESD circuit during the high current or high voltage ESD transient stage [7]. This paper presents a PTSCR that investigates the change of the electrical characteristics of the device under high temperature conditions for various process technologies.

\section{PTSCR description review}

The cross-sectional view and equivalent of the proposed device is shown in Fig. 1. This device is made by adding a 2-finger-ggNMOS structure and $\mathrm{p}+$ tab onto a conventional SCR with an $\mathrm{n}+$ bridge diffusion across the $\mathrm{n} / \mathrm{p}$-well junction. The 2-finger ggNMOS is formed on the right-hand side of the device to reduce the trigger voltage and to generate the trigger current. The $\mathrm{p}+\mathrm{tab}$ is formed at the center of the conventional SCR and connected to the center diffusion (source of the ggNMOS) of the ggNMOS. The $\mathrm{p}+$ tab provides the p-well on the left-hand side of the device with the self-trigger current generated by the ggNMOS. A parasitic diode is imbedded in the structure. In negative stress mode, the device operates as a diode to discharge the ESD current. As the pad voltage increases, the potential of the n-well as well as the drains of the ggNMOS stack increase.

Eventually, the drains of the ggNMOS stack and the p-well junction begin to avalanche because the high electric field crosses it, subsequently generating electron-hole pairs. The holes drift toward the p-substrate contact and strengthen a potential of the p-well, and the potential from the center diffusion of the ggNMOS stack to the substrate junction increases and gives forward biases at this junction. Two parasitic npn bipolar transistors turnon on and the current 'Path1' is formed, as shown in Fig. 1. This trigger 


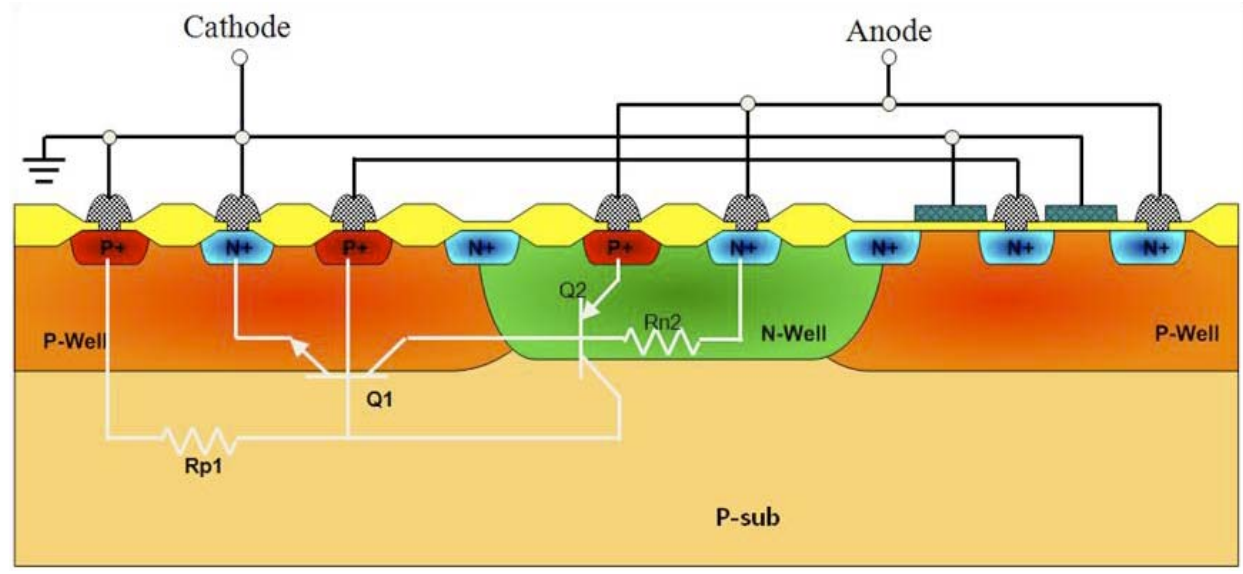

Fig. 1. Cross-sectional and equivalent circuit view of the PTSCR

current flows toward the $\mathrm{p}+$ cathode through the $\mathrm{p}+$ tab/p-well. As the trigger current increases, the $\mathrm{p}$-well $/ \mathrm{n}+$ cathode junction forward biases. The parasitic npn bipolar (collector: $\mathrm{n}+/ \mathrm{n}$-well anode, base: $\mathrm{p}$-well, emitter: $\mathrm{n}+$ cathode) and the parasitic pnp bipolar (collector: p+ anode, base: n-well, emitter: p+ cathode) turn on. Then, the mostly discharged current passes through 'Path 2', as shown in Fig. 1. On the other hand, when a negative bias is applied on the pad with a grounded line, the parasitic diode ( $\mathrm{p}+$ cathode/p-well or p-substrate $/ \mathrm{n}$-well $/ \mathrm{n}+$ anode) in the proposed device will be forward biased to discharge the negative ESD current [6].

\section{Experimental result}

PTSCR devices were successfully implemented in a $0.13 \mathrm{um}, 0.18$ um CMOS technology and in a $0.35 \mathrm{um}$ BCD technology. The $\mathrm{I}-\mathrm{V}$ characteristics and DC-leakage current were obtained with a Transmission Line Pulse (TLP) tester with square pulse duration of $100 \mathrm{~ns}$ and a default pulse rise time of $10 \mathrm{~ns}$. Also, temperature characteristics of the device were measured with a TLP tester using a HOT CHUCK controller.

A. Electrical characteristic: The results of the TLP measurements for the PTSCR in each process technology are depicted in Fig. 2. The holding voltage and trigger current of the devices in each process are $5.7 \mathrm{~V}(0.13 \mathrm{um}), 7.2 \mathrm{~V}$ (0.18 um), $8 \mathrm{~V}(0.35 \mathrm{um})$ and $121 \mathrm{~mA}(0.13 \mathrm{um}), 144 \mathrm{~mA}(0.18 \mathrm{um}), 103 \mathrm{~mA}$ $(0.35 \mathrm{um})$, respectively. Due to the MOS trigger mechanism, the trigger current of PTSCR $(>100 \mathrm{~mA})$ is greater than that of the other ESD devices (Generally $<1 \mathrm{~mA}$ ). Also, because of the reduction of the parasitic NPN current gain by the high trigger current, its holding voltage is higher than other SCR based ESD devices.

B. Temperature characteristics: In order to analyze the temperature dependency of the PTSCR, measurements were performed at various temperatures $(300 \mathrm{~K}-500 \mathrm{~K})$.

Fig. 3 shows the results of the trigger current/holding voltage at certain temperature ranges. At an elevated temperature, the holding voltage of the 


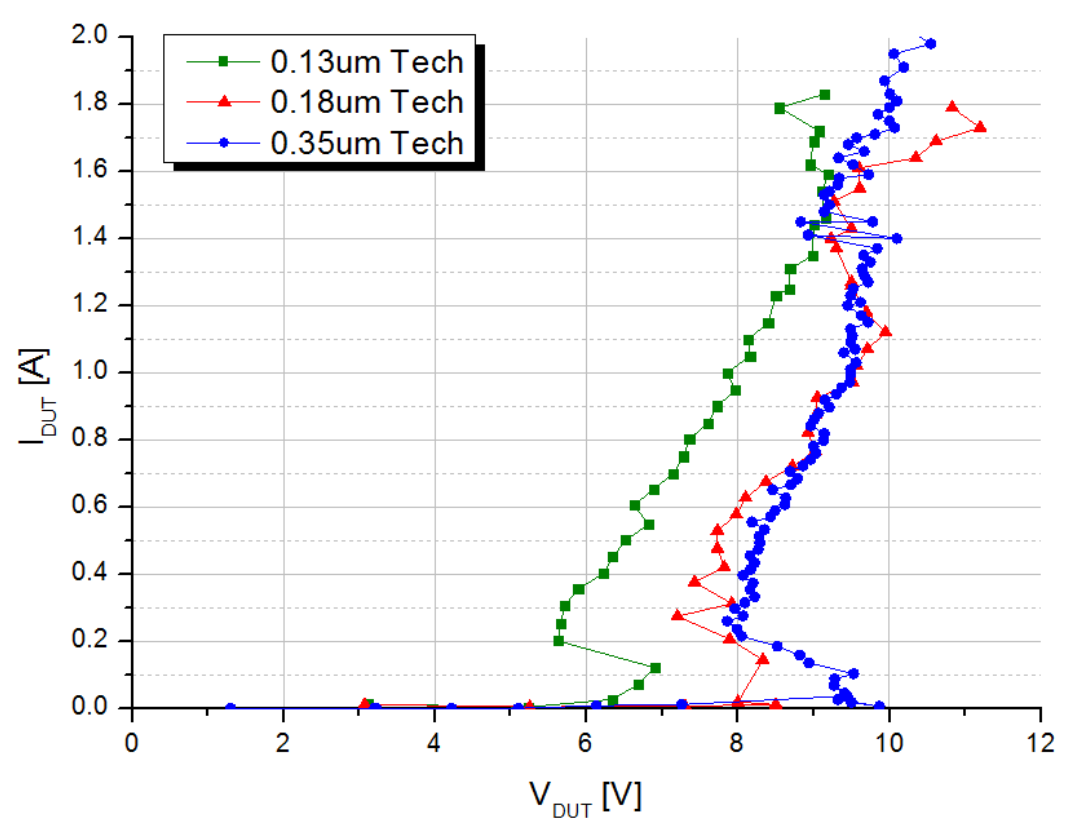

Fig. 2. TLP I-V characteristics of PTSCR in $0.13 \mathrm{um}$, $0.18 \mathrm{um}, 0.35 \mathrm{um}$ process technology

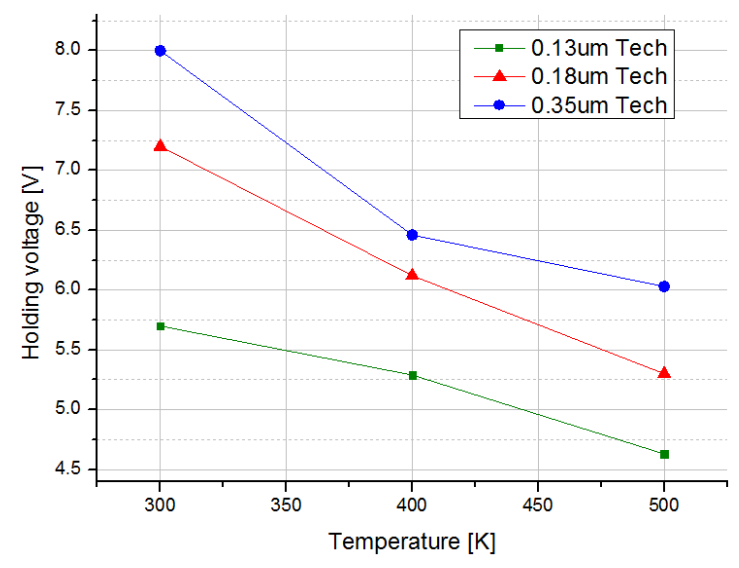

(a)

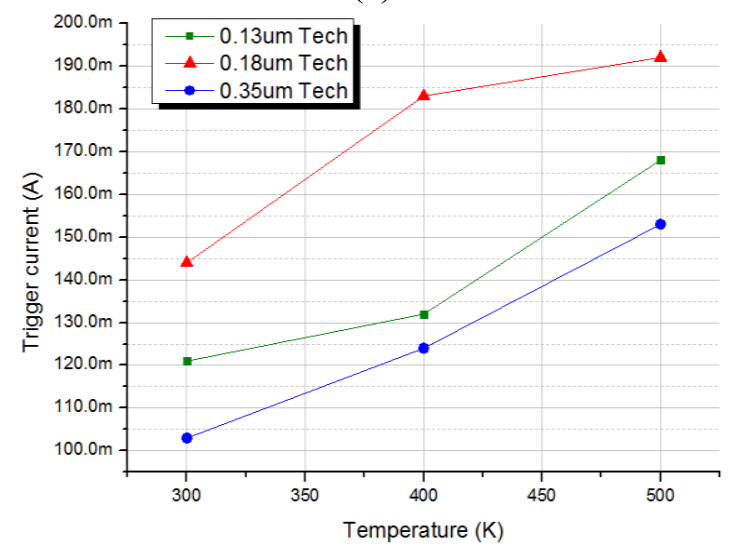

(b)

Fig. 3. Thermal measurement of (a) holing voltage and (b) trigger current in $0.13 \mathrm{um}, 0.18 \mathrm{um}, 0.35 \mathrm{um}$ process technology 
PTSCR is reduced by parasitic bipolar transistors. The holding voltage is the sum of VEB (Q2) and VCE (Q1) (refer to Fig. 1). By increasing the temperature, VEB (Q2) decreased while VCE (Q1) remained almost constant. Therefore, the holding voltage of the SCR decreases in high temperatures. On the contrary, the trigger current increased with the increase of temperature. The trigger mechanism of the PTSCR is equal to it of the MOSFET. At elevated temperatures, the mean free path of the MOSFET possessed a much shorter distance than the mean free path in room temperature. So, the avalanche current shows an increase due to the generated impact ionization in strong electric fields. Therefore the trigger current increases.

\section{Conclusion}

This paper investigated the electrical characteristics of SCR based ESD protection devices (PTSCR) in various process technologies. This structure contained a high holding voltage and a high trigger current in each process technology. These characteristics help avoid latch-up in normal operating conditions. Also, we found that the holding voltage decreased and trigger current increased with increasing temperature. By increasing the temperature, the holding voltage is dominated by the main SCR structure and the trigger current is mainly governed by GGNMOS situated in right-hand side of the structure.

\section{Acknowledgments}

The present research was conducted by the research fund of Dankook University in 2010 . 